\title{
Towards a Service-Oriented Architecture for Interactive Ubiquitous Entertainment Systems
}

\author{
Giovanni Cagalaban and Seoksoo Kim ${ }^{*}$ \\ Department of Multimedia, Hannam University, Daejeon, Korea \\ gcagalaban@yahoo.com, sskim0123@naver.com
}

\begin{abstract}
Ubiquitous computing is not only applied to doing daily activities and integrated into everyday objects but for entertainment, and gaming as well. In this research, we explore the relevance of ubiquitous computing to entertainment systems using devices such as mobile devices. We introduce a service-oriented architecture for ubiquitous entertainment systems to establish collaborative relationships between heterogeneous devices to provide users an interactive and ubiquitous entertainment and fun.
\end{abstract}

Keywords: ubiquitous entertainment, service-oriented architecture, mobile device.

\section{Introduction}

The vision of having computing services readily available is becoming more of a reality [1][2]. Ubiquitous computing has also applied in ubiquitous entertainment systems [3]. The emergence of mobile devices and the recent trend in $3 \mathrm{G}$ technology enables people to work, play games, or entertain themselves at home, office, or even on the move [4]. The capability of wireless devices to connect to a combination of networks such as wireless fidelity (WiFi), worldwide interoperability for microwave access (WiMAX), global system for mobile communication (GSM), and Bluetooth connections allows them to interoperate in a heterogeneous environment.

In this research, we design a service-oriented architecture for ubiquitous entertainment systems to establish collaborative relationships between heterogeneous devices. The proposed service oriented architecture enables mobile users to find themselves in environments rich in ubiquitous services that their mobile devices can take advantage of for entertainment, gaming, and fun.

\section{Ubiquitous Entertainment Services}

The ubiquitous service center has more computing power compared to the mobile devices that people use. Examples of such ubiquitous environment may be in a conference room, coffee shop, restaurant, train station, airport, or shopping mall, etc. The ubiquitous service centers can be configured in controlled and relatively stable environments, such as homes, offices, or public areas that experience a high density

\footnotetext{
Corresponding author.
} 
of mobile users to provide ubiquitous services such as shown in Fig. 1 where a user is playing a game in a mobile device surrounded by sensor network and is equipped with situation awareness to seamlessly perform other activities [5].

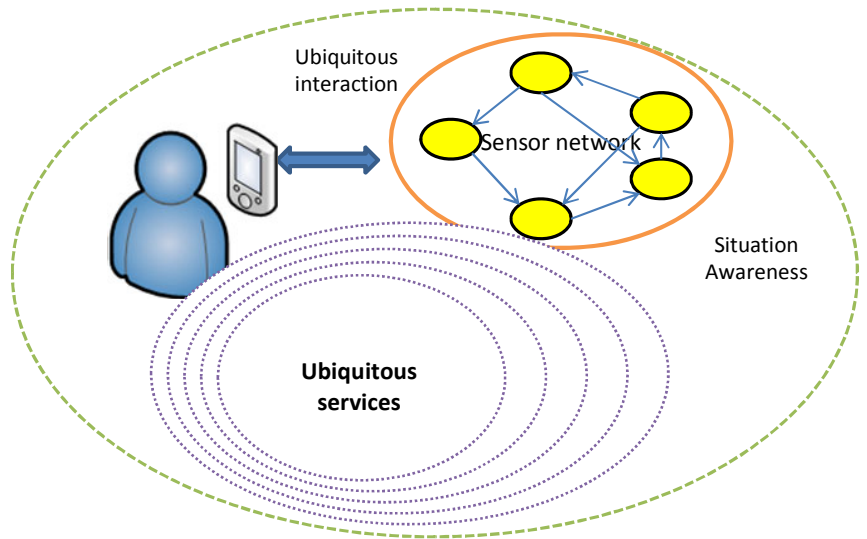

Fig. 1. Ubiquitous environment

In the ubiquitous entertainment system, a mobile user should be able to access and consume the services provided by the ubiquitous entertainment server through the ubiquitous environment in a seamless manner. The proposed service oriented architecture for the ubiquitous entertainment system is shown in Fig. 2.

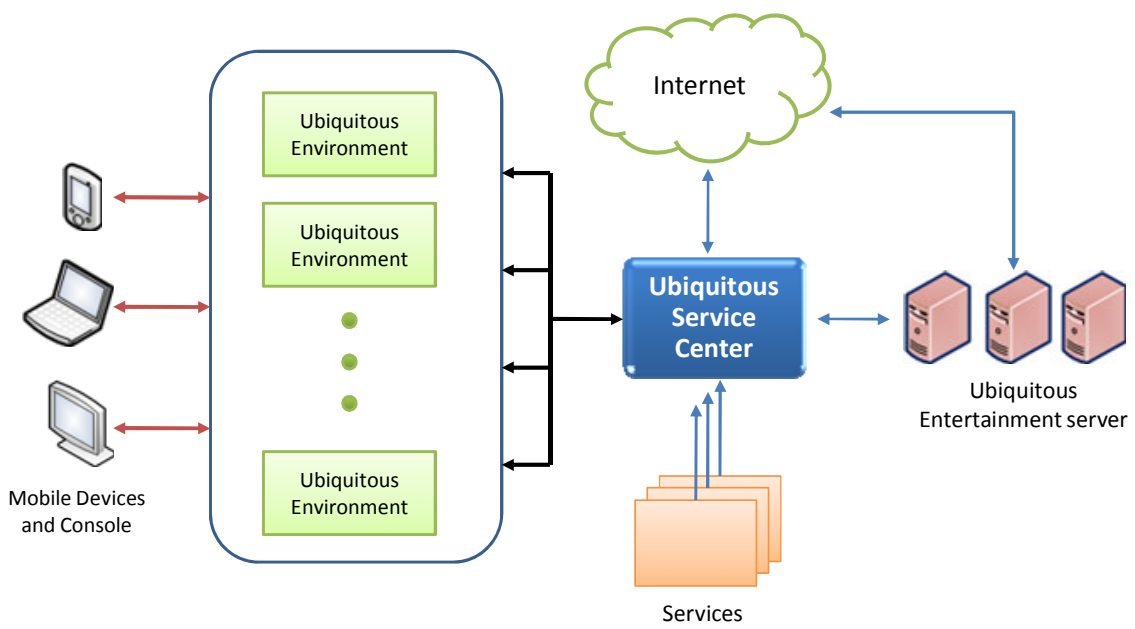

Fig. 2. Service oriented architecture for ubiquitous entertainment system

One of the functions of the ubiquitous service center is to register and query of services. The registration and discovery of services in a ubiquitous service center is done by the service manager. The service manager is responsible for facilitating the 
registration service where mobile devices can discover and select from a set of services. Also, ubiquitous service center is responsible for session management. If a user of a mobile device discovers that there are available services in the ubiquitous service center that are of his interest, he can query and request the service manager for services to be used by his mobile device.

In the service oriented architecture of the ubiquitous entertainment system, the main focus of the design is the service interface. We consider that all the service components are deployed in ubiquitous environment and are visible for mobile devices to invoke or consume over the network. Also, the nature of SOA is focused on creating services using components with well defined interfaces, which allows devices to be loosely coupled with each other.

\section{Conclusion}

We have presented the significance of introducing a service-oriented architecture for ubiquitous entertainment system. The main focus of the service oriented architecture is the creation of flexible and extensible interfaces for the interactive delivery of services for a ubiquitous entertainment. The design of a service-oriented architecture is an integral part of ubiquitous technology. In the future, we aim to improve the security design of the ubiquitous entertainment system by implementing security algorithms to enhance reliability and improved performance of ubiquitous services.

\section{Acknowledgements}

This paper has been supported by the 2010 Hannam University Research Fund.

\section{References}

1. Weiser, M.: The computer for the twenty-first century. Scientific American 265(3), 94-104 (1991)

2. Davies, N., Gellersen, H.W.: Beyond prototypes: Challenges in deploying ubiquitous systems. IEEE Pervasive Computing 1, 26-35 (2002)

3. Endres, C., Butz, A., MacWilliams, A.: A Survey of Software Infrastructures and Frameworks for Ubiquitous Computing. Mobile Information Systems Journal 1(1), 41-80 (2005)

4. Cheok, A.D., Fong, S.W., Goh, K.H., Yang, X., Liu, W., Farzbiz, F.: Human Pacman: A Sensing-based Mobile Entertainment System with Ubiquitous Computing and Tangible Interaction. In: Proceedings of the Second Workshop on Network and System Support for Games (2003)

5. Sung, J., Lopez, T.S., Kim, D.: The EPC sensor network for RFID and WSN integration infrastructure. In: IEEE International Conference on Pervasive Computing and Communications Workshops, Los Alamitos, USA, pp. 618-621 (2007) 